LAWRENCE LIVERMORE N A TION AL LABORATORY

\title{
Electron-Cloud Effects on Heavy-Ion Beams
}

R.H. Cohen, A. Friedman, T. Azevedo, J.-L. Vay

March 31, 2004 
This document was prepared as an account of work sponsored by an agency of the United States Government. Neither the United States Government nor the University of California nor any of their employees, makes any warranty, express or implied, or assumes any legal liability or responsibility for the accuracy, completeness, or usefulness of any information, apparatus, product, or process disclosed, or represents that its use would not infringe privately owned rights. Reference herein to any specific commercial product, process, or service by trade name, trademark, manufacturer, or otherwise, does not necessarily constitute or imply its endorsement, recommendation, or favoring by the United States Government or the University of California. The views and opinions of authors expressed herein do not necessarily state or reflect those of the United States Government or the University of California, and shall not be used for advertising or product endorsement purposes.

This work was performed under the auspices of the U.S. Department of Energy by University of California, Lawrence Livermore National Laboratory under Contract W-7405-Eng-48. 


\title{
Electron-Cloud Effects on Heavy-Ion Beams
}

\author{
R. H. Cohen, ${ }^{*}$ A. Friedman, and S. M. Lund \\ Lawrence Livermore National Laboratory, \\ P.O. Box 808, Livermore, CA 94550 \\ T. Azevedo and J.-L. Vay \\ Lawrence Berkeley National Laboratory, Berkeley, CA 94720
}

(Dated: March 30, 2004 DRAFT)

\begin{abstract}
Stray electrons can be introduced in positive-charge accelerators for heavy ion fusion (or other applications) as a result of ionization of ambient gas or gas released from walls due to haloion impact, or as a result of secondary-electron emission. We are developing a capability for self-consistent simulation of ion beams with the electron clouds they produce. We report on an ingredient in this capability, the effect of specified electron cloud distributions on the dynamics of a coasting ion beam. We consider here electron distributions with axially varying density, centroid location, or radial shape, and examine both random and sinusoidally varying perturbations. We find that amplitude variations are most effective in spoiling ion beam quality, though for sinusoidal variations which match the natural ion beam centroid oscillation or breathing mode frequencies, the centroid and shape perturbations can also be effective. We identify a possible instability associated with resonance with the beam-envelope "breathing" mode. One conclusion from this study is that heavy-ion beams are surprisingly robust to electron clouds, compared to a priori expectations.
\end{abstract}

*rcohen@llnl.gov 


\section{INTRODUCTION}

Stray electrons are becoming increasingly recognized as a significant issue for heavy-ion fusion (HIF) accelerators. Indeed, such "electron clouds" have long been recognized as a serious issue for some other accelerator applications, such as high-energy physics [? ]. The common thread in these contexts is that the electron cloud is an uncontrolled source of negative charge that can lead to alteration of the ion beam dynamics, possibly leading to beam deflection, increased beam emittance, envelope size, and halo, and also potentially driving electron-ion instabilities. For an application such as heavy-ion fusion where there is an economic imperative to have the ion beam fill as much of the cross section of the beam line as possible, electron cloud effects can be a significant factor in limiting this beam pipe fill factor.

The Heavy-Ion Fusion Virtual National Laboratory (HIF-VNL) is developing a capability for self-consistent simulation of electron clouds and their effect on ion beams.[? ] This activity entails adding, to the WARP simulation code [? ], models describing emission of secondary electrons, neutral gas, and scattered ions when beam ions strike the wall, as well as models for the subsequent transport and ionization of neutrals and an efficient ion-timescale description of electron dynamics.

A key question is, how much electron density is required to produce a substantial perturbation in ion beam quality? The completed code capability described above will enable a quantitative assessment; as an interim indicator, we have undertaken a series of WARP ion simulations with prescribed (i.e., non-self-consistent) electron clouds. Besides providing an indication of the tolerable level of electron contamination, these simulations also shed light on the types of electron perturbations that would be of greatest concern. A preliminary version of this work was reported in ref. [? ]; we present here revised and more extensive results.

\section{PROBLEM SETUP}

These studies consist of a series of WARP slice simulations (an $x-y$ slice of ions is followed in the axial coordinate $z$ ) in which we have added a specified, frozen-in-time, negative charge distribution which represents the electron cloud. This non-dynamical model of the electrons 
is useful not only for providing estimates of tolerable electron levels, but also for illuminating important electron-ion interaction mechanisms, as will be seen.

For heavy-ion induction accelerators, in contrast to accelerators for high-energy physics, the dominant source of electrons is likely to be ionization of neutral gas released at walls, as opposed to secondary electrons. There are several reasons for this: (1) The relatively long pulse length gives time for neutrals to penetrate into the ion beam and for significant ionization of the neutrals; (2) The electrons resulting from ionization of gas are born trapped in the beam's electrostatic potential well (in contrast to secondary electrons born at the wall), and so are confined for a long time; and (3) In the absence of special wall-scrubbing techniques (difficult to apply for induction accelerators), experiments have shown that the neutral-particle emission per incident ion is much higher than the secondary-electron emission $\left(\sim 10^{3}-10^{5}\right.$ versus $\left.\sim 10-100\right)[? ?]$, more than enough of a ratio to overcome the relatively low $\left(10^{-2}-10^{-1}\right)$ probability of ionization of the neutrals during the beam pulse.

This observation has a substantial impact on the type of model to use in our studies. Because induction accelerators have a beam pipe that runs through the quadrupole magnets but is absent in the gaps, and because the elliptical distortion of the ion beam is maximum in the quadrupoles, beam-ion loss occurs almost exclusively well within quadrupole magnets. Electrons born in such a circumstance are strongly magnetized (gyroradius $\rho_{e}$ much smaller than the transverse beam dimensions) and so tend to follow magnetic field lines, with small electric and magnetic drifts across the field. Since the tips of the elliptical beam are in regions of field lines that are nearly tangent to the beam pipe and never penetrate far into the plasma interior (see Fig 1), electrons emitted as a result of impact of primary beam ions on the wall are in general trapped close to the wall, and remain there until they drift out of the quadrupole magnet. There will be a relatively small population of electrons which reach the beam interior, as a result of some ions scattering from the beam pipe hitting the beam pipe again on field lines which penetrate the beam interior, and as a result of electrons drifting out of a quadrupole magnet and ceasing to be tied to field lines (the drift time being of order the pulse duration). But even those two groups of electrons will tend to produce the highest densities near the beam pipe, as their velocities are smallest there. Hence clouds produced by secondary-electron production will tend to be hollow.

In contrast, if the pulse duration is long enough that neutrals released from the walls penetrate deep into the beam interior, then electrons born from ionization of these neutrals 
will have a density distribution that is sizeable where the ion beam density is large. Also, electrons born from ionization of ambient gas will fall within the beam cross section. For constant neutral density, the electron source function would just be proportional to the ion density; the actual electron density would not be, because of the subsequent electron bounce motion in the combined magnetic and potential wells. But certainly the electron density would be concentrated within the beam envelope.

For any of the mentioned electron sources, the electron density will be large within quadrupoles compared to that between quadrupoles, due to magnetic confinement: the beam-wise drift motion of electrons within a quadrupole is slow compared to the beam-wise thermal motion of electrons between quadrupoles.

In light of the above considerations our base model electron distribution is chosen to be a constant charge density transverse to the beam direction out to the (elliptical) beam envelope boundary, and axially constant within the nominal axial extent of a quadrupole magnet, but zero outside of these limits. The base case also has the constant charge density the same in each quadrupole. The beam envelope is calculated in the absence of any electron cloud. We run ion beam simulations with this base model, and with a number of perturbations added to this distribution. These are: (i) electron density varying from quadrupole to quadrupole (but constant within a quadrupole); (ii) centroid of the electron cloud displaced by an amount which is constant within a quadrupole but varies from quadrupole to quadrupole; (iii) addition of a radially parabolically varying (zero-volume-integrated) density contribution, $\delta n_{e} \sim$ const $\times\left[(x / a)^{2}+(y / b)^{2}-1 / 2\right]$ where $a$ and $b$ are the envelope semi-axes (versus $z$ ), and the constant, which can be positive or negative, is constant within a quadrupole; this perturbation allows the electron cloud to be peaked or hollow; and (iv) addition of an additional stretch along one axis and shrink along the other of the electron cloud envelope, by a factor that is (again) constant in a quadrupole but varying from quad to quad. We consider two types of axial variations: (a) random from quadrupole to quadrupole; and (b) sinusoidally varying from quadrupole to quadrupole (except that we have not done random perturbations of the elliptical distortion, i.e. type iv). In the case of the centroid variation, for the random cases we let the direction of displacement also be random; for sinusoidal cases, the centroid rotates (with fixed fractional displacement relative to the envelope) as one progresses from quadrupole to quadrupole.

Each of the types of perturbations represents a component of the variability we can 
expect in accelerators, as there can be differing amounts and shape of halo, ion-beam centroid displacements, and shifts in the relative amounts of electrons from gas ionization versus from secondary emission, due to variations in wall conditions, magnet or beam misalignments, and natural oscillations of the beam. The types of random variations are representative of what might be expected from random alignment errors and random changes in wall conditions. The sinusoidal variations are chosen to explore electrons from, and possible resonance with, breathing, centroid oscillation, and quadrupole oscillation modes of the beam.

All simulations are run for a $2 \mathrm{MeV}$ Potassium beam through 100 alternating gradient lattice periods of a model beam transport system (no acceleration). Each magnet is identical, and represented by gridded field data from a 3D magnet calculation. The magnets are 10.2 $\mathrm{cm}$. long, have a radial field gradient of $90.51 \mathrm{~T} / \mathrm{m}$. The gaps alternate in length between $6.1 \mathrm{~cm}$ and $18.5 \mathrm{~cm}$ ("syncopated lattice"), giving a total lattice period of $45 \mathrm{~cm}$. The beam is taken to be bounded by a circular-cross-section beam pipe, of radius $2.95 \mathrm{~cm}$. Simulation particles that reach the pipe wall are simply removed from the computation. A list of beam, lattice and simulation parameters is given in Appendix A.

For this case of no acceleration, the spatial frequencies for the various beam modes (centroid oscillations, breathing mode, quadrupole oscillations) are easily derived in a continuousfocus approximation; the frequencies for these modes are defined and evaluated, including the effects of the electron space charge, in Appendix B. For the sinusoidal cases we vary the frequency in the vicinity of the nominal resonant frequency to search for a resonance with the beam mode.

\section{RESULTS}

In our base case, the electron density in each quadrupole is constant within the beam envelope, and the constant density is the same from quadrupole to quadrupole. The magnets are tuned for a matched beam with no electrons. We show, in Fig. 2, results (an $x-y$ scatter plot, emittance of beam fractions, envelope, and beam current versus axial position $z$ ) for the base case with electron density $n_{e} 20 \%$ of the beam density $n_{b}$. Considering the size of the electron density, there is surprisingly little beam degradation: a small growth in the emittance, but virtually no envelope growth or beam loss.

Figs. 3-5 show the results for various types of random perturbations. For all cases, 
the random component is equal in peak magnitude to the constant component; hence the electron density for the $20 \%$ mean case varies from zero to $40 \%$ of the beam (ion) density); for axial displacements, the centroid varies from zero to the nominal envelope half width; for radial shape variations, the maximum radial density variation is from zero to double the nominal electron density. These are, obviously, extreme variations. For all cases the shown $x-y$ scatter plot represents a "typical bad slice" chosen from near the end of the simulation. The choice of which plot to show is arbitrary; we pick a case with a relatively large, and in some sense interesting, halo. As is evident, the random amplitude case is the most effective in degrading the beam; a $20 \%$ mean electron density leads to a loss of $10 \%$ of the beam over the 100-quadrupole system. Examination of the set of plots in Fig. 3 indicates that the major incidents of beam loss are associated with periods where the beam envelope is especially large and in fact close to the wall, and also that the emittance grows at about the same $z$ locations, but the growth is limited to the outermost class of ions.

Figs 4 and 5 indicate that random offsets and random beam shape variations are considerably less effective than random amplitude variations in degrading beam quality, especially for the outermost ions. One might have supposed that these sorts of perturbations would be particularly effective in coupling to centroid oscillations and breathing modes of the beam, but apparently this coupling is not particularly effective for random perturbations. As we see below, this is in marked contrast to the situation with a sinusoidally varying perturbation.

Next we turn to the sinusoidally varying cases. Fig. 6 shows the fraction of beam loss versus perturbation wavelength, normalized to the lattice period. This is for sinusoidally (versus quadrupole number) varying density, with a 5\% mean electron density. We see that there is a sharp maximum, close to the nominal breathing mode resonance, corresponding to a beam loss of $28 \%$. We notice that the loss is comparable to the $20 \%$ mean randomly varying amplitude case. Fig. 7 shows the $x-y$ scatter, emittance growth, envelope growth, and beam current at the peak of the resonance curve of Fig. 6.

Figures 8 and 9 show the corresponding quantities for a radially varying electron cloud shape (still resonant with breathing modes). In this case the mean electron density is $10 \%$ of the ion beam density. Again there is a sharp maximum versus perturbation frequency. We notice that the maximum beam loss for this case is smaller than that for amplitude variations (even at the larger mean electron density), but is still larger than any of the random variations studied. 
Figures 10 and 11 show the corresponding quantities for when the electron cloud centroid is varied sinusoidally at frequencies in the vicinity of that for centroid oscillations. Again, the electron density in the cloud is $10 \%$. Once again a resonance is found, with a peak beam current loss about half that for the radial shape variation resonance, and still larger than any of the random variations at comparable electron density.

Figures 12 and 13 show the corresponding quantities for factor of two variations in the ellipticity, again for electron density $10 \%$ of the beam density. Here, the resonance is with quadrupolar distortion modes of the ion beam. Again there is a distinct resonance, but the effect on the ion beam, as measured by beam loss, is the weakest of the resonant interactions considered - only around 4\%. On the other hand the effect on the emittance is significant, and it penetrates farther in from the edge of the beam; this is in marked contrast to all of the other perturbations.

We note that, for sinusoidal amplitude variations, there was no discernible resonance when the frequency was scanned through the range of the centroid and quadrupole resonances. This is in marked contrast to the observations of Figs 5 and 6. This is expected: the transverse-plane symmetry of the amplitude variation implies that this perturbation cannot couple to centroid oscillations or quadrupole oscillations, but can effectively couple to breathing modes.

Finally, we examine the phase of the electron cloud and the ion beam distortions that it produces. We show in Figure 14 an overlay of plots of the beam envelope and the electron density for the sinusoidally varying case. We see that, through much of the early growth of the beam envelope, the two curves are in phase (the peaks coincide). Later, they drift out of phase, perhaps because the beam breathing mode changes frequency as beam current is lost.

\section{DISCUSSION}

The results presented here indicate that electrons are most detrimental to ion beam quality when the amplitude varies from quadrupole to quadrupole, and a density variation that is resonant with beam breathing modes is considerably more effective than a random variation in producing beam loss and degradation of beam quality. For a 100 lattice-period system, a mean 5\% electron population, with a 100\% resonant modulation, can lead to loss 
of 28 percent of the beam. For random variations, even a 10\% mean electron density, still with $100 \%$ modulation, (not shown) leads to loss of only about $2 \%$ of the beam; the $20 \%$ case shown in Fig. 3 results in a $10 \%$ beam loss. Random variations in electron cloud shape or centroid are considerably less effective. Resonant variations in electron cloud shape (resonating with the breathing mode) or in centroid position (resonating with centroid oscillations) are effective in degrading beam quality, but for the perturbations tested not as effective as resonant amplitude variations.

An interesting result, and potentially a significant concern, is the phase relationship between the electron perturbation and the envelope oscillations shown in Fig 14. The density variation and the beam envelope are approximately in phase until the beam envelope is large enough that the beam current has significantly changed. This in-phase relationship could give rise to an instability: if there is a resonant component to the electron cloud (a seed perturbation), then the beam envelope will tend to be biggest where the electron cloud is biggest, resulting in the biggest beam scrape-off there, and hence the biggest source of electrons. The electrons so produced are rather well confined axially by the magnetic field; hence they will stay in the region where they are produced, adding to the initial perturbation. The next slice of the beam to pass through will see an amplified electron perturbation, leading to a yet larger localized loss. This mechanism can operate even if the ion beam is accelerated so that the spatial frequency is not constant in space: so long as there is a component of the electron density that matches the (spatially varying) beam envelope oscillations, that component will grow because of axial localization of the electrons. Effects that will limit growth include beam velocity "tilt" (the variation in mean axial velocity along the beam from head to tail), electron drift out of a quadrupole, and change of the resonant frequency due to beam loss. A similar instability mechanism could operate for the other resonant perturbations (shape variations, centroid oscillations, quadrupolar distortions), as well. We will report on an analytic model of this instability and a search for it in self-consistent electron-ion simulations, in the future.

It is evident from examination of the $x-y$ scatter plots, even the few shown, that the nature of the beam degradation is more complex than is captured in the line plots of emittance, envelope, and beam current. In particular, we see from Fig 3a a beam whose core is nearly circular but which has a strongly elliptical (and in fact reaching the wall) halo. The same beam, further along, has a core that is elliptical but has a nearly circular halo 
(Fig. 15). The elliptical distortion of the main beam would suggest ion scrape-off only on field lines that are nearly tangent to the wall. However, from this latter plot we see that the halo resulting from the electron cloud can be much more symmetric, implying scrape-off on field lines that penetrate deep into the beam interior. This would lead to a relatively larger role for secondary electrons than one would otherwise infer. From the sequence of the two scatter plots, we infer that, not unexpectedly, the ions in the halo are experiencing different oscillation frequencies from those in the core. In Fig. 7a, a different phenomenon is occurring, leading to halo that is actually concentrated (at this instant) near the quadrupole field lines that penetrate deep into the plasma interior.

There are several overall conclusions to be drawn from this study: (1) Heavy-ion beams are actually surprisingly robust to electron clouds. A priori there was some expectation that even a few tenths of a percent electrons would be devastating. Even for resonant sinusoidally varying electron clouds with $100 \%$ modulation, electron densities in the range of several percent of the beam density are required for substantial beam degradation. A constant (from quadrupole to quadrupole) electron density, such as might be caused by ionization of the base pressure of background gas, results in negligible beam loss and envelope growth, and only very minor emittance growth; (2) Electron cloud density modulation resonant with the beam breathing mode is the most dangerous kind of perturbation (at least based on beam loss) and may well be the basis for an instability; instability growth will be limited by beam velocity tilt, electrons drifting out of quadrupoles, and frequency shift resulting from beam loss; (3) elliptical distortions which resonate with beam quadrupole modes have a global effect on beam emittance (related to the effect of a beam mismatch), even though the beam current loss they produce is relatively modest, and (4) electron cloud impact on ion beam propagation is a rich and complex subject, and is not well characterized simply by the evolution of a few beam moments. 
APPENDIX A: BEAM, LATTICE AND SIMULATION PARAMETERS.

\begin{tabular}{|l|l|}
\hline parameter & value \\
\hline mass & $38.9637 \mathrm{amu}$ (Potassium) \\
charge & +1 \\
initial current & $810 \mathrm{~mA}$ \\
initial emittance (unnormalized) & $100 \mathrm{~mm} . \mathrm{mrad}$ \\
initial perveance & $1.1565 \times 10^{-3}$ \\
energy & $2 \mathrm{MeV}$ \\
initial dimensions & $a_{0}=b_{0}=1.17344 \mathrm{~cm}$ \\
initial divergences & $a_{0}^{\prime}=-b_{0}^{\prime}=-34.616 \mathrm{mrad}$ \\
initial distribution & $\mathrm{semi}$ gaussian \\
undepressed phase advance & $80^{\circ}$ \\
depressed phase advance & $19.8^{\circ}$ \\
lattice period & $45 \mathrm{~cm}$ \\
No. of lattice periods & 100 \\
quad. lattice occupancy & 0.453 \\
quad. syncopation factor & 0.248 \\
field gradient & $90.51 \mathrm{~T} / \mathrm{m}$ \\
pipe radius & $2.948 \mathrm{~cm}$ \\
No. of simulation grid cells & $256 \times 256$ \\
No. of macroparticles & 400000 \\
\hline
\end{tabular}

APPENDIX B: WAVENUMBERS OF THE NATURAL MODES OF OSCILLATIONS FOR CHARGED PARTICLE BEAMS IN A FODO LATTICE, INCLUDING THE EFFECT OF NEUTRALIZATION.

For the study of oscillations of the envelope of a beam in a periodic lattice [? ? ], the relevant beam parameters are its perveance $K$ and its emittance $\varepsilon$. The perveance, a dimensionless constant which can be interpreted as the ratio of potential energy $W_{f}$ due to the beam self-field to kinetic energy $W_{k}$ for a beam particle traveling on the edge, is given by 


$$
K=\frac{W_{f}}{W_{k}}=\frac{q I /\left(4 \pi \varepsilon_{0} v_{z}\right)}{1 / 2 m v_{z}^{2}}=\frac{q I}{2 \pi \varepsilon_{0} m v_{z}^{3}}
$$

where $q$ and $m$ are respectively the charge and mass of the beam particles, $v_{z}$ is the average axial beam velocity, $I$ is the beam current and $\varepsilon_{0}$ is the permittivity of vacuum. The emittance is a measure of the phase space area of the beam. It can also be thought of as a measure of beam quality. In ideal accelerator lattices, it should be nearly constant.

In the smooth focusing approximation, the mean values of the beam envelope functions $X(z)$ and $Y(z)$ follow the equations

$$
\begin{aligned}
& X^{\prime \prime}+k_{0}^{2} X-\frac{2 K}{X+Y}-\frac{\varepsilon^{2}}{X^{3}}=0, \\
& Y^{\prime \prime}+k_{0}^{2} Y-\frac{2 K}{X+Y}-\frac{\varepsilon^{2}}{Y^{3}}=0,
\end{aligned}
$$

where $k_{0}$ is the wavenumber for a single particle oscillating in the lattice and is related to the lattice quadrupoles strength and geometry. $k_{0}$ is also the natural wavenumber of oscillations for the beam centroid.

For a matched beam, one can rewrite $\mathrm{X}$ and $\mathrm{Y}$ as a function of the mean radius $\bar{R}$ which is a constant

$$
\begin{aligned}
& X(z)=\bar{R}\left(1+\delta_{x}(z)\right), \\
& Y(z)=\bar{R}\left(1+\delta_{y}(z)\right) .
\end{aligned}
$$

Assuming $\delta_{x}(z) \ll \bar{R}$ and $\delta_{y}(z) \ll \bar{R}, \bar{R}$ obeys the equation

$$
k_{0}^{2} \bar{R}-\frac{K}{\bar{R}}-\frac{\varepsilon^{2}}{\bar{R}^{3}}=0
$$

An analysis of Eqs. (??) and (??) shows that the oscillations of $X(z)$ and $Y(z)$ can always be decomposed in two fundamental modes whose wavenumbers are given by

$$
\begin{aligned}
& k_{b}=\sqrt{2 k_{0}^{2}+2 k^{2}} \\
& k_{q}=\sqrt{k_{0}^{2}+3 k^{2}}
\end{aligned}
$$

with

$$
k=\sqrt{k_{0}^{2}-\frac{K}{\bar{R}^{2}}}
$$

The first mode $k_{b}$, called the "breathing" mode, corresponds to the case where the oscillations in $X$ and $Y$ are in phase, while the second mode $k_{q}$, called the "quadrupole" mode, 
corresponds to the case where those oscillations are $180^{\circ}$ out of phase. $k$ can be interpreted as the wavenumber of single particle oscillations in the core of the beam in response to applied focusing and space-charge forces. The inequality $0 \leq k / k_{0} \leq 1$ provides a normalized measure of space-charge strength with $k / k_{0}=1$ corresponding to zero space-charge and $k / k_{0}=0$ to the space-charge limit.

If a uniform neutralized background is added, i.e. electrons, with neutralization fraction $f=n_{e} / n_{i}$, where $n_{e}$ and $n_{i}$ are respectively the electron and ion densities, then the perveance of the beam is reduced by the factor $(1-f)$ and Eqs. (??), (??) and (??) become respectively

$$
\begin{gathered}
k^{*}=\sqrt{k_{0}^{2}-\frac{K(1-f)}{\bar{R}^{2}}}, \\
k_{b}^{*}=\sqrt{2 k_{0}^{* 2}+2 k^{* 2}}, \\
k_{q}^{*}=\sqrt{k_{0}^{* 2}+3 k^{* 2}},
\end{gathered}
$$

with

$$
k_{0}^{*}=\sqrt{k_{0}^{2}+\frac{S^{2} f K}{\bar{R}^{2}}} .
$$

where $S$ is the lattice period. Note that the electron density has been treated as an external perturbation.

In our case, we have $K \sim 1.1565 \times 10^{-3}, \varepsilon \sim 10^{-4} \mathrm{~m} . \mathrm{rad}, S=0.45 \mathrm{~m}, \sigma_{0}=k_{0} S=80^{\circ}$, where $\sigma_{0}$ is the undepressed phase advance. Solving (??) gives an average radius of $\bar{R} \sim 1.13$ $\mathrm{cm}$. Scaled wavenumbers for the two fundamental modes are given in table ?? for four values of the neutralization factor. This indicates that the wavenumber of both modes should increase with higher electron density. We observe this trend in our scans of frequency for the sinusoidal perturbations. For the case of amplitude modulations, which excites the breathing mode, the peak of beam loss was around $k_{b} S \sim 0.333$ for an average $f=2.5 \%$ and around $k_{b} S \sim 0.34$ for an average $f=5 \%$. For the case of shape modulation, which also triggers the breathing mode, the peak of beam loss was around $k_{b} S \sim 0.342$ for an average $f=10 \%$. For the case of 'squeeze-stretch' modulation, which triggers the quadrupole mode, the peak of beam loss was around $k_{b} S \sim 0.254$ for an average $f=10 \%$.

In all cases, the observed wavenumbers were significantly above the one computed for no electron density, as predicted. The observed values are remarkably close to the predictions in the case of amplitude modulation, which might be partially coincidental since the theory was in the smooth limit while the simulation involves beams departing significantly from this 
TABLE I: Scaled wavenumber for the two fundamental modes of a beam in a periodic lattice for four neutralization factors.

\begin{tabular}{|c|c|c|c|c|}
\hline $\mathrm{f}(\%)$ & 0. & 2.5 & 5. & 10. \\
\hline \hline$k_{b} S$ & 0.324 & 0.331 & 0.338 & 0.351 \\
\hline$k_{q} S$ & 0.242 & 0.252 & 0.261 & 0.278 \\
\hline
\end{tabular}

limit. For the case of centroid displacement of the electron density, which triggers oscillations of the beam centroid, the peak of beam loss has been observed around $k_{o} S \sim 0.207$ for an average $f=10 \%$, below the value of $k_{0} S \sim 0.222$ for $f=0$, in apparent contradiction with the formula (??) which predicts larger values of $k_{0}$ for larger values of $f$. However, the assumption of focusing effect of the electron density on the beam which led to the multiplication of the perveance by the factor $(1-f)$ does not hold in this case. An offset electron density precessing around the axis has an overall defocusing effect on the beam since it will pull the beam off axis, in effect reducing the wavenumber of the natural mode of oscillation.

\section{ACKNOWLEDGMENTS}

This work was performed under the auspices of the U.S. Department of Energy by Lawrence Livermore National Laboratory under contract W7405-ENG-48 and by Lawrence Berkeley National Laboratory under contract DE-AC03-76F00098.

[] See e.g., ECLOUD 02 Workshop, CERN Report CERN-2002-001, http:// wwwslap.cern.ch/collective/ecloud02/proceedings/

[] R. H. Cohen, A. Friedman, M.A. Furman, S.M. Lund, A.W. Molvik, P. Stoltz, J.-L. Vay, "Stray-Electron Accumulation and Effects in HIF Accelerators", Particle Accelerator Conference, Portland OR, 2003, paper TOAA010; http://epaper.kek.jp/p03/INDEX.HTM

[] D. P. Grote, A. Friedman, I. Haber, "Three-Dimensional Simulations of High Current Beams in Induction Accelerators with WARP3d," Fus. Eng. \& Des., 32-33, 193 (1996) and http://hif.lbl.gov/theory/WARP_summary.html 
[] A. Faltens, private communication (2001).

[] A. W. Molvik, D. Baca, F.M. Bieniosek, R.H. Cohen, A. Friedman, E.P. Lee, S.M. Lund, L. Prost, A. Sakumi, P.A. Seidl, J.L. Vay, "Initial Experimental Studies of Electron Accumulation in a Heavy-Ion Beam", Particle Accelerator Conference, Portland OR, 2003, paper TOPC004; http://epaper.kek.jp/p03/INDEX.HTM

[] M. Reiser, "Theory and design of charged particle beams", John Wiley \& Sons, Inc., New-York (1994)

[] J. J. Barnard and S. M. Lund, "Space charge effects in beam transport", course notes, U.S. Particle Accelerator School, Boulder, Colorado (2001), and private communication (2004) 


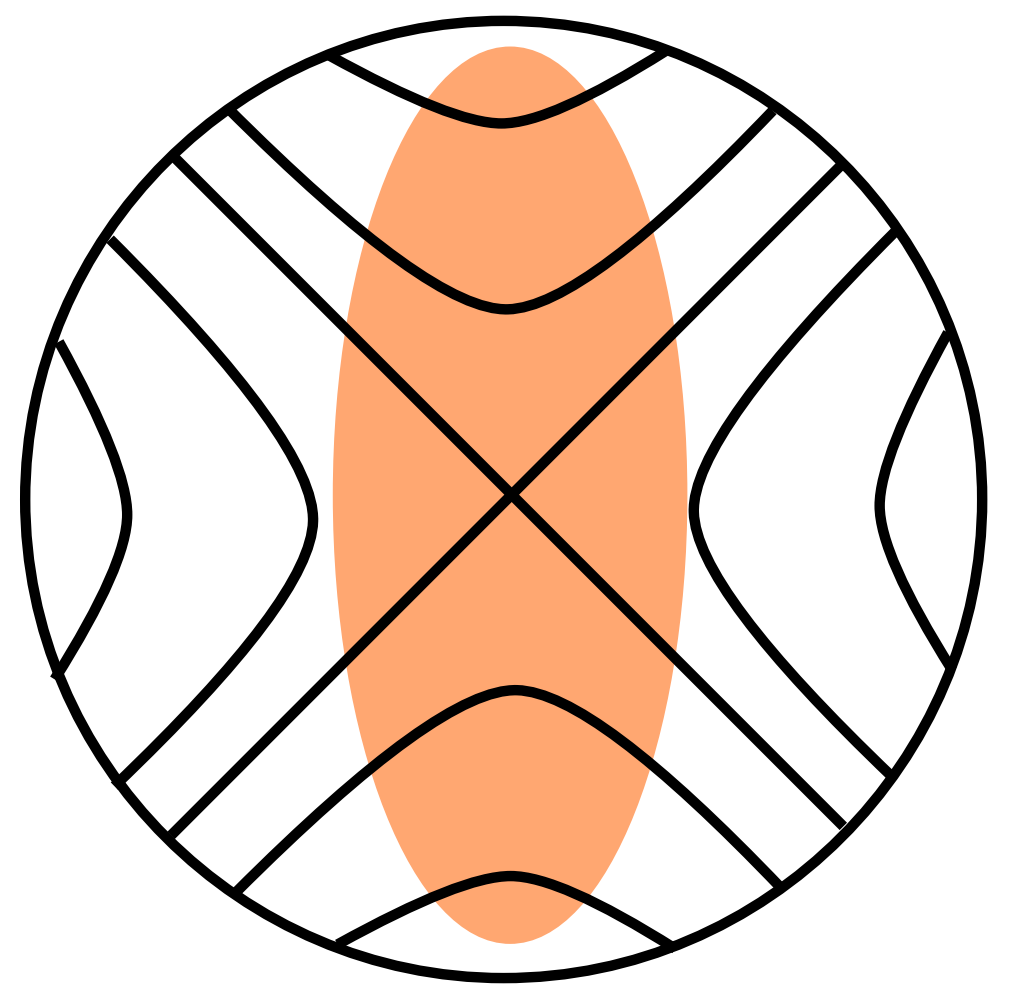

FIG. 1: Sketch of magnetic field lines and elliptical beam envelope in a quadrupole magnet 

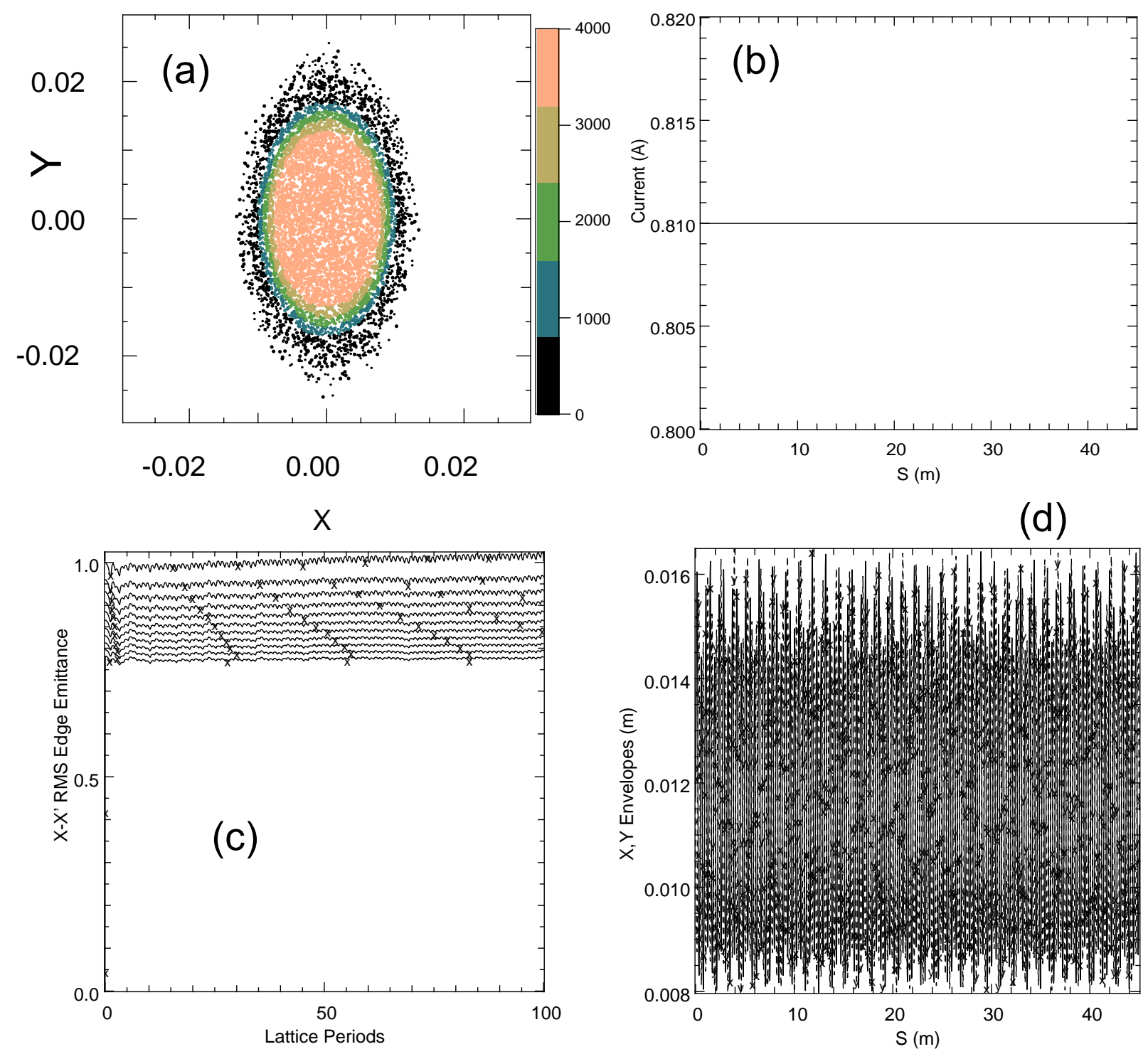

FIG. 2: Results for fixed $20 \%$ electron density in each magnetic quadrupole: (a) $x-y$ scatter plot of ions near end of magnet array; (b) beam current versus axial position; (c) emittance in $x$ plane versus axial position for various percentages of the beam current enclosed by nested ellipses in phase space (90\% to $100 \%$ in $1 \%$ increments); (d) $x$ and $y$ envelope semi-axes (the RMS extents times two) versus axial position 

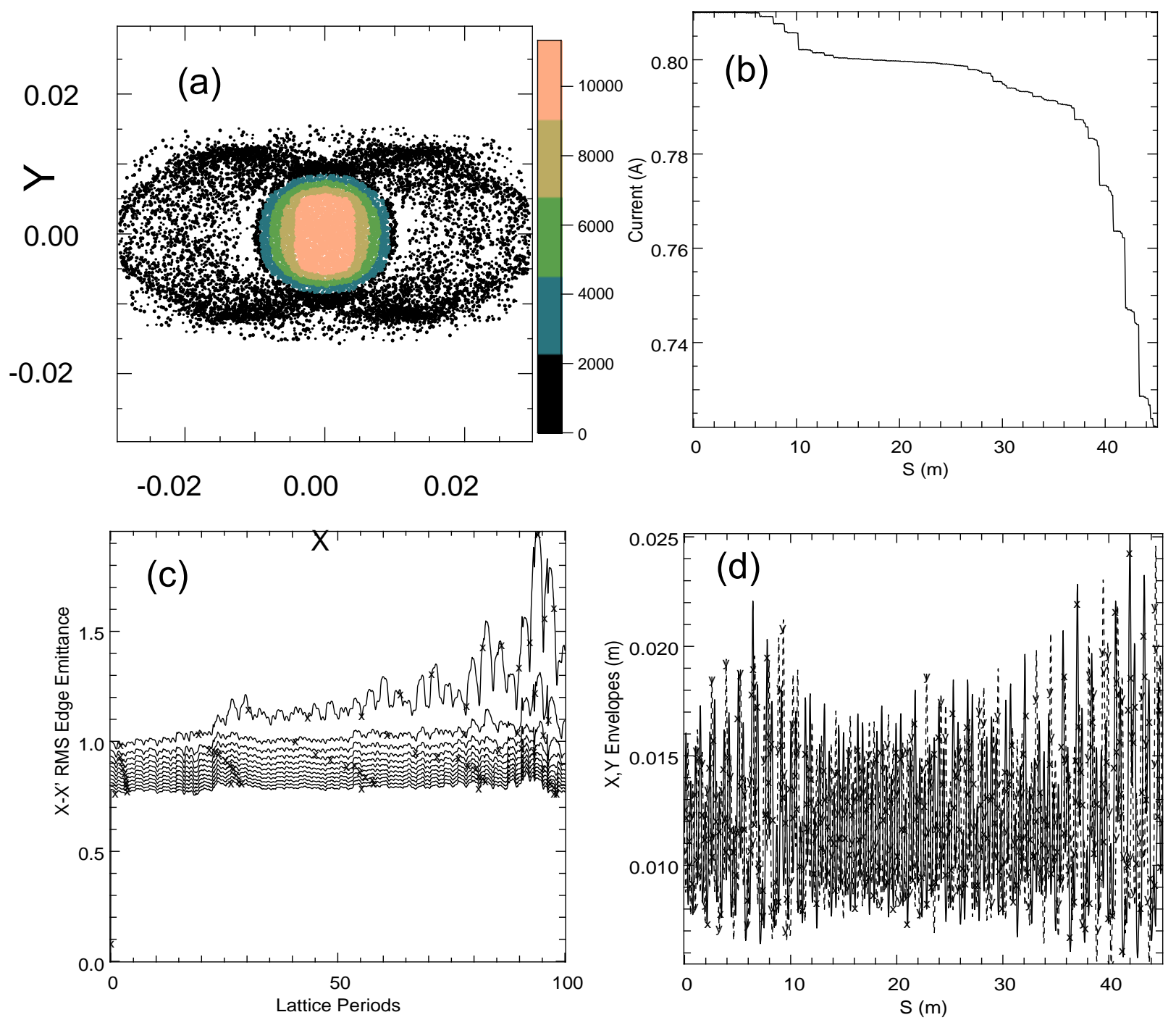

FIG. 3: Results for random amplitude fluctuations, with $20 \%$ mean electron density and $100 \%$ modulation. (a)-(d) have same significance as in Fig. 2. 

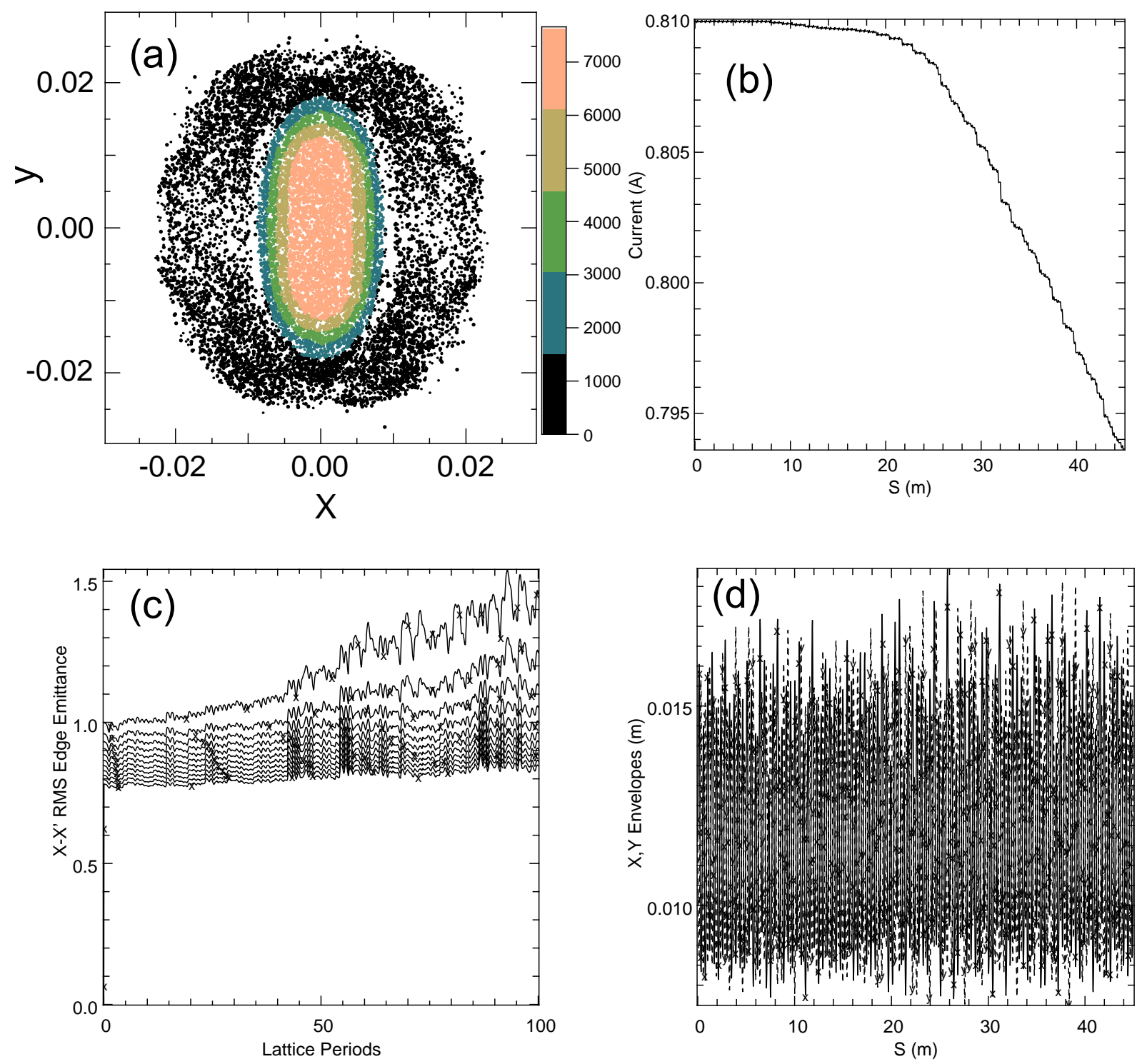

FIG. 4: Results for random electron-cloud centroid shifts, with $20 \%$ mean electron density and shifts out to $100 \%$ of the envelope radius. (a)-(d) have same significance as in Fig. 2. 

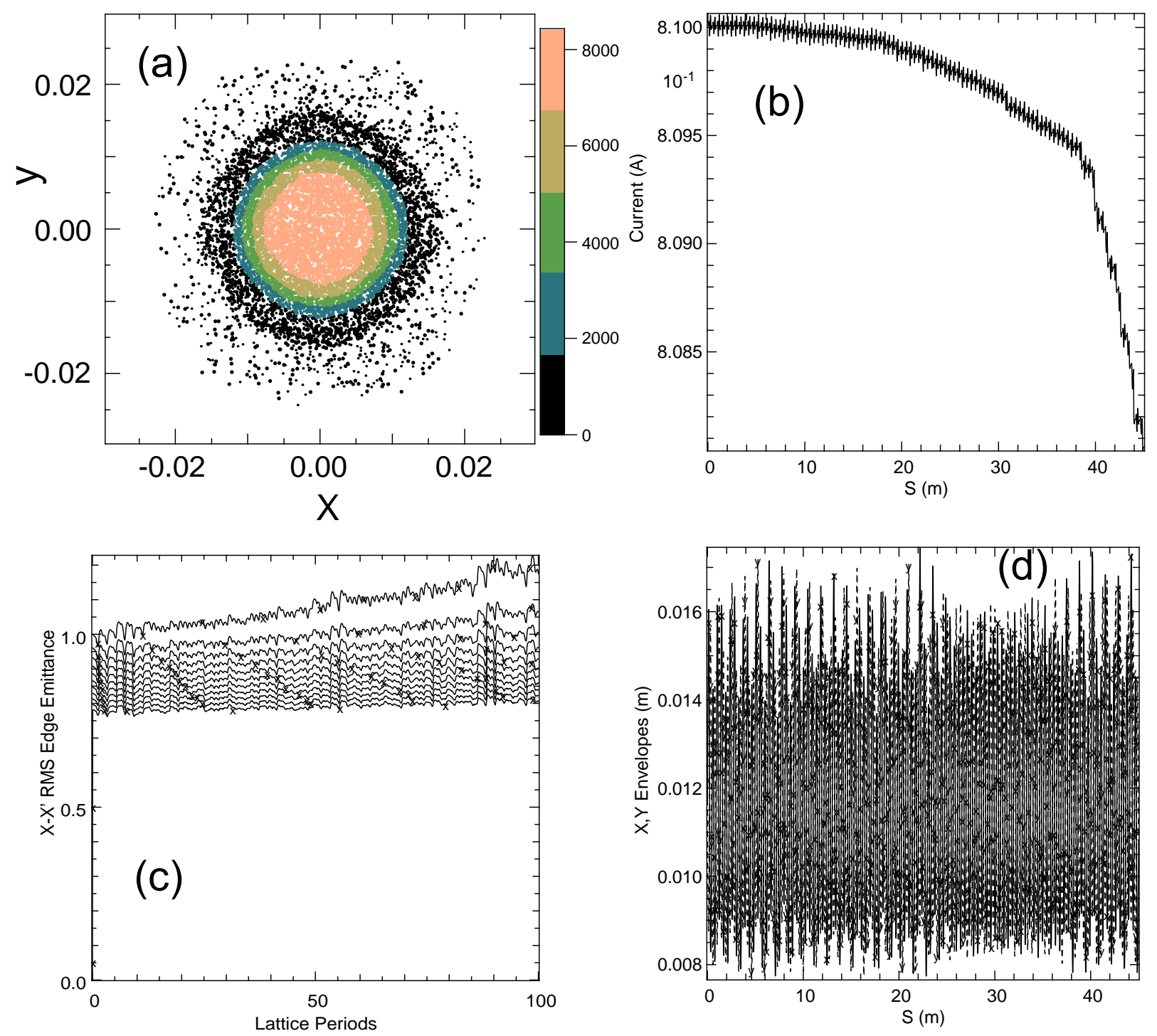

FIG. 5: Results for random electron-cloud radial shape variations, with $20 \%$ mean electron density and $100 \%$ modulation (radial density variation from as little as 0 to as much as twice the mean): (a)-(d) have same significance as in Fig. 2. 


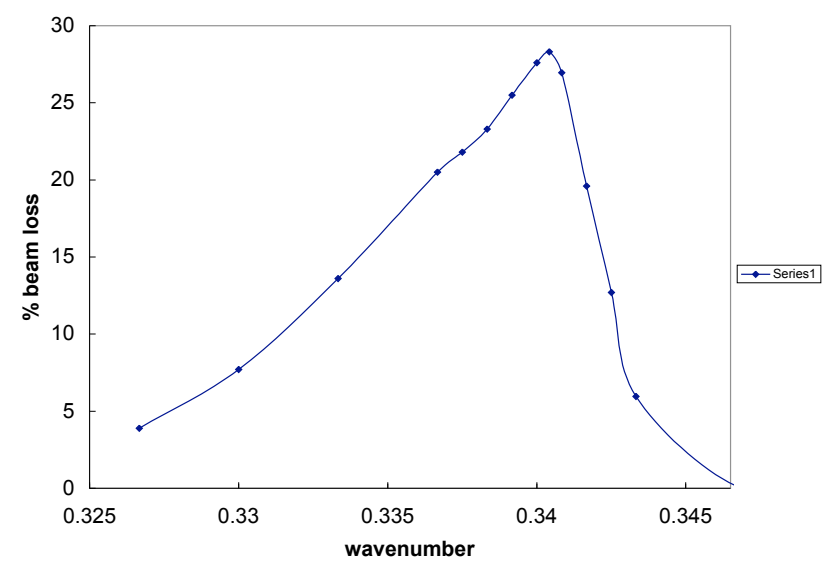

FIG. 6: Fractional beam loss versus perturbation wavenumber for electron cloud density that varies sinusoidally with quadrupole number. Mean electron density is $5 \%$ of central beam density and modulation is $100 \%$. Wavenumber is normalized to inverse of lattice period. 

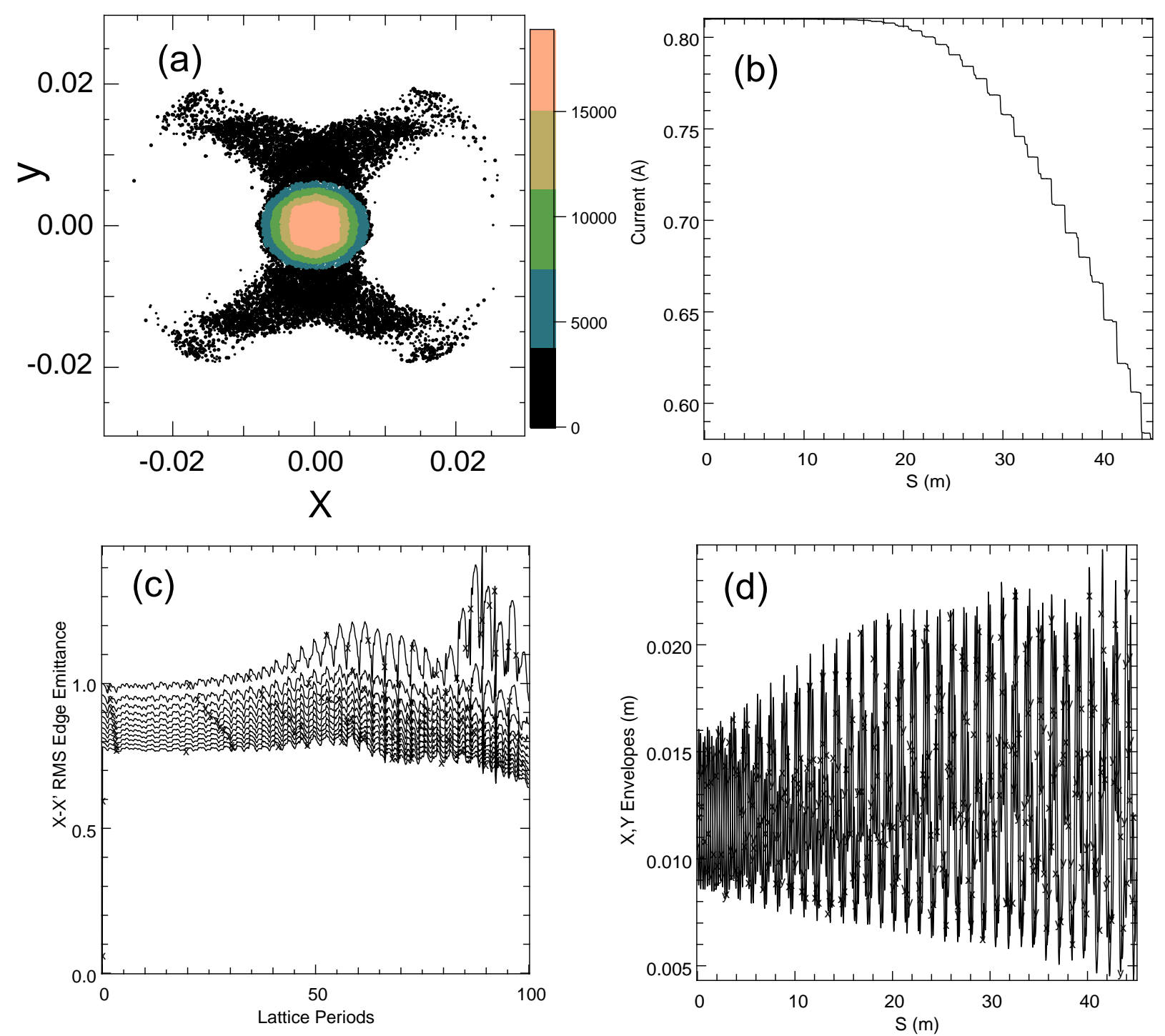

FIG. 7: Results for sinusoidally varying electron density at the peak in Fig. 6. (a)-(d) have same significance as in Fig. 2. 


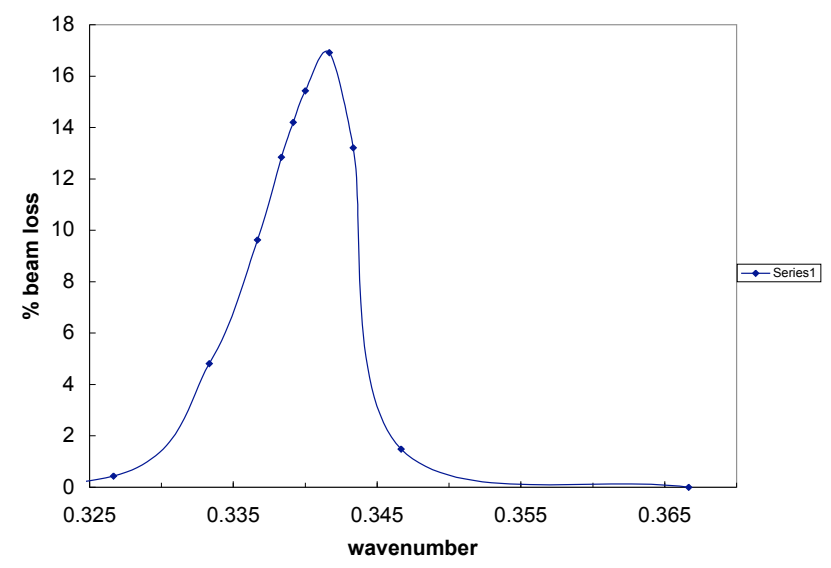

FIG. 8: Fractional beam loss versus perturbation wavenumber for electron cloud radial shape that varies sinusoidally with quadrupole number. Electron density has a mean of $10 \%$ of central beam density and at peak of sine varies from 0 to twice the mean. Wavenumber is normalized to inverse of lattice period. 

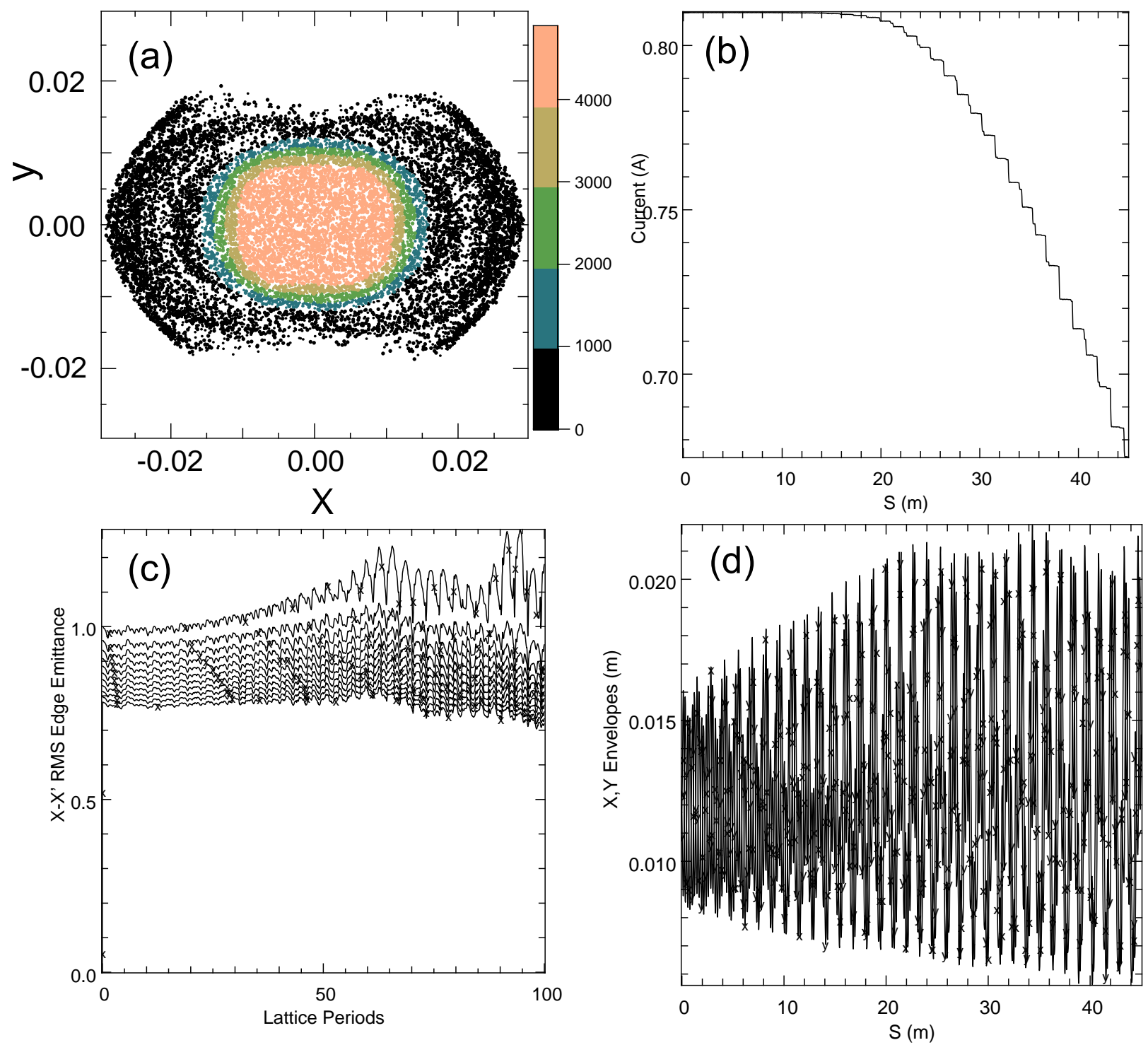

FIG. 9: Results for sinusoidally varying radial shape of electron density at the peak in Fig. 8. (a)-(d) have same significance as in Fig. 2. 


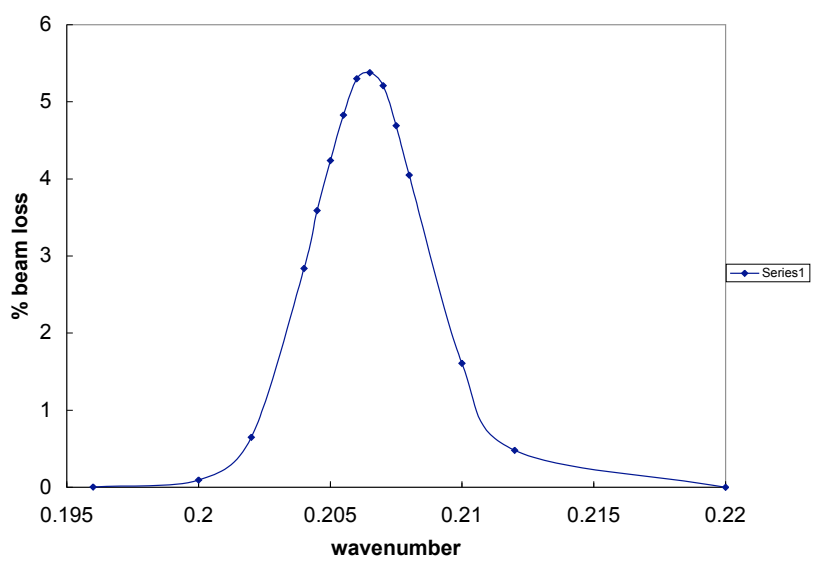

FIG. 10: Fractional beam loss versus perturbation wavenumber for electron cloud offset that rotates sinusoidally with quadrupole number. Electron density is $10 \%$ of central beam density and offset is local envelope radius. Wavenumber is normalized to inverse of lattice period. 

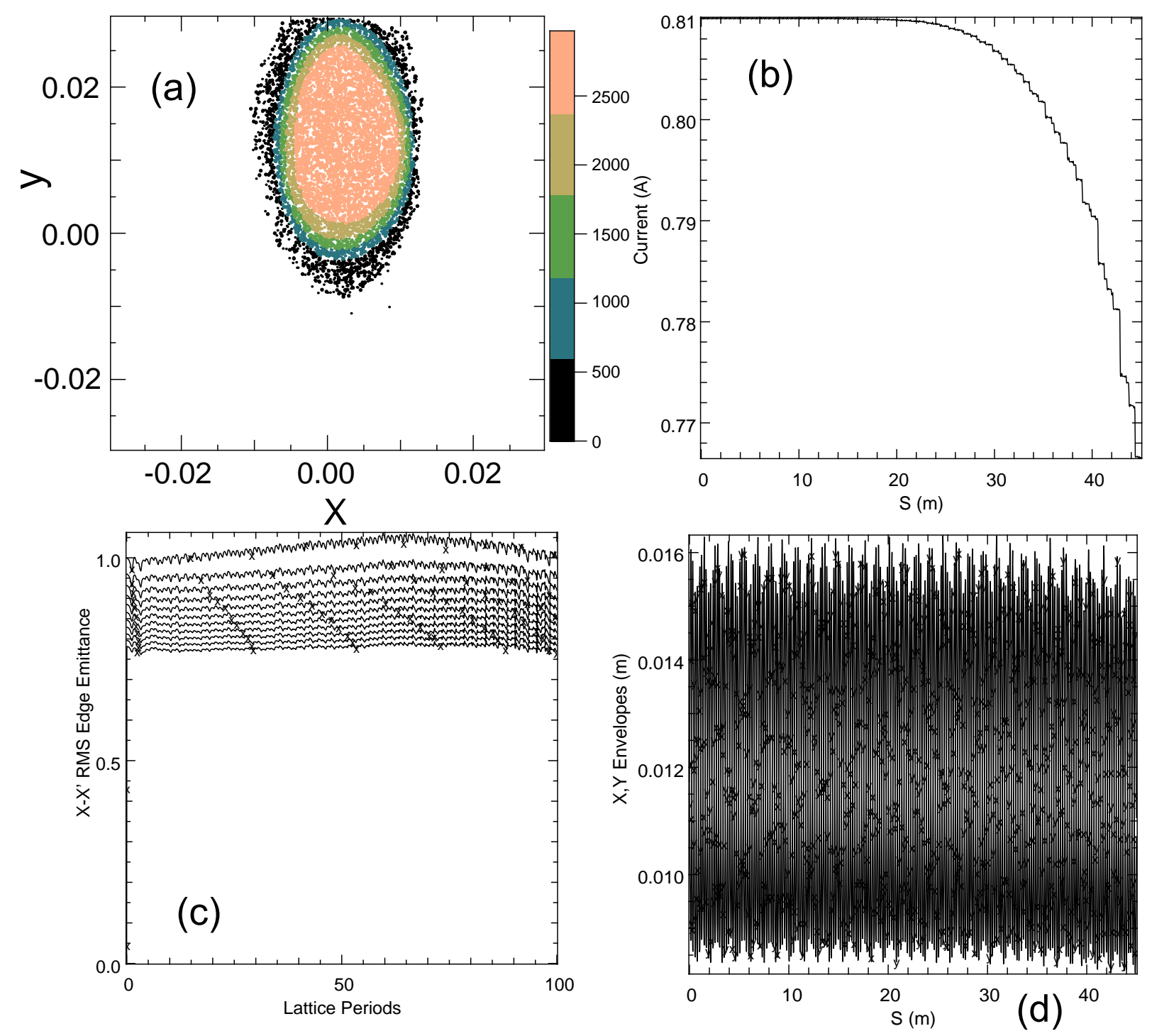

FIG. 11: Results for sinusoidally varying centroid position at the peak in Fig. 10. (a)-(d) have same significance as in Fig. 2. 


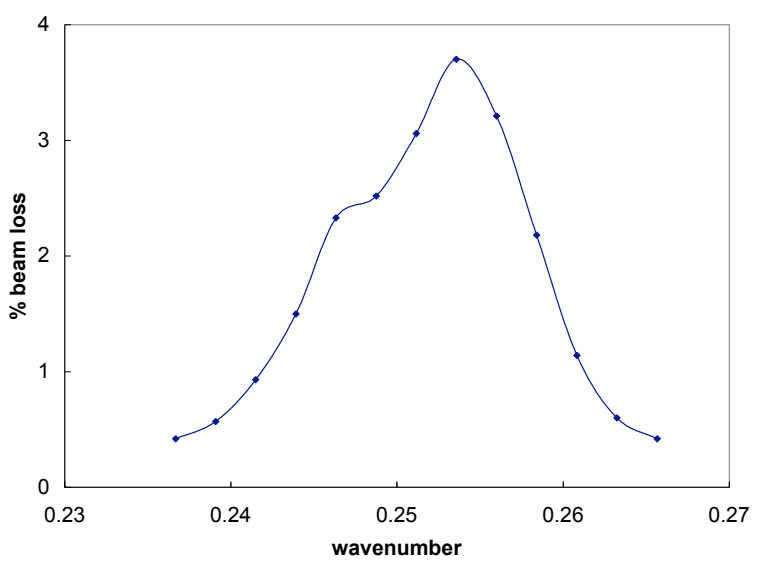

FIG. 12: Fractional beam loss versus perturbation wavenumber for electron cloud elliptical distortion that varies sinusoidally with quadrupole number. Electron density is $10 \%$ of central beam density. Maximum enhancement or degradation of ellipticity is a factor of two. Wavenumber is normalized to inverse of lattice period. 

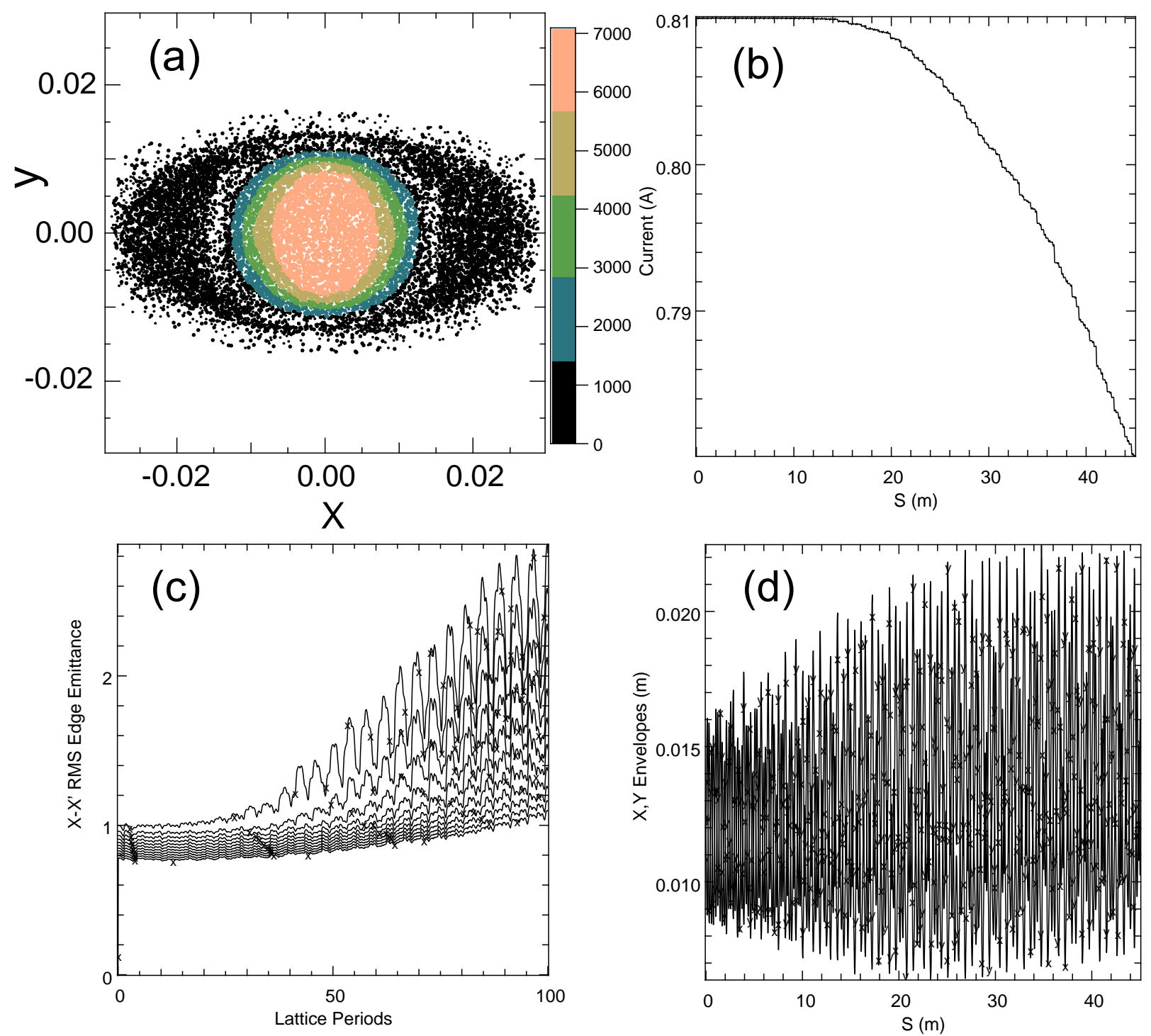

FIG. 13: Results for sinusoidally varying ellipticity of electron cloud at the peak in Fig. 12. (a)-(d) have same significance as in Fig. 2. 


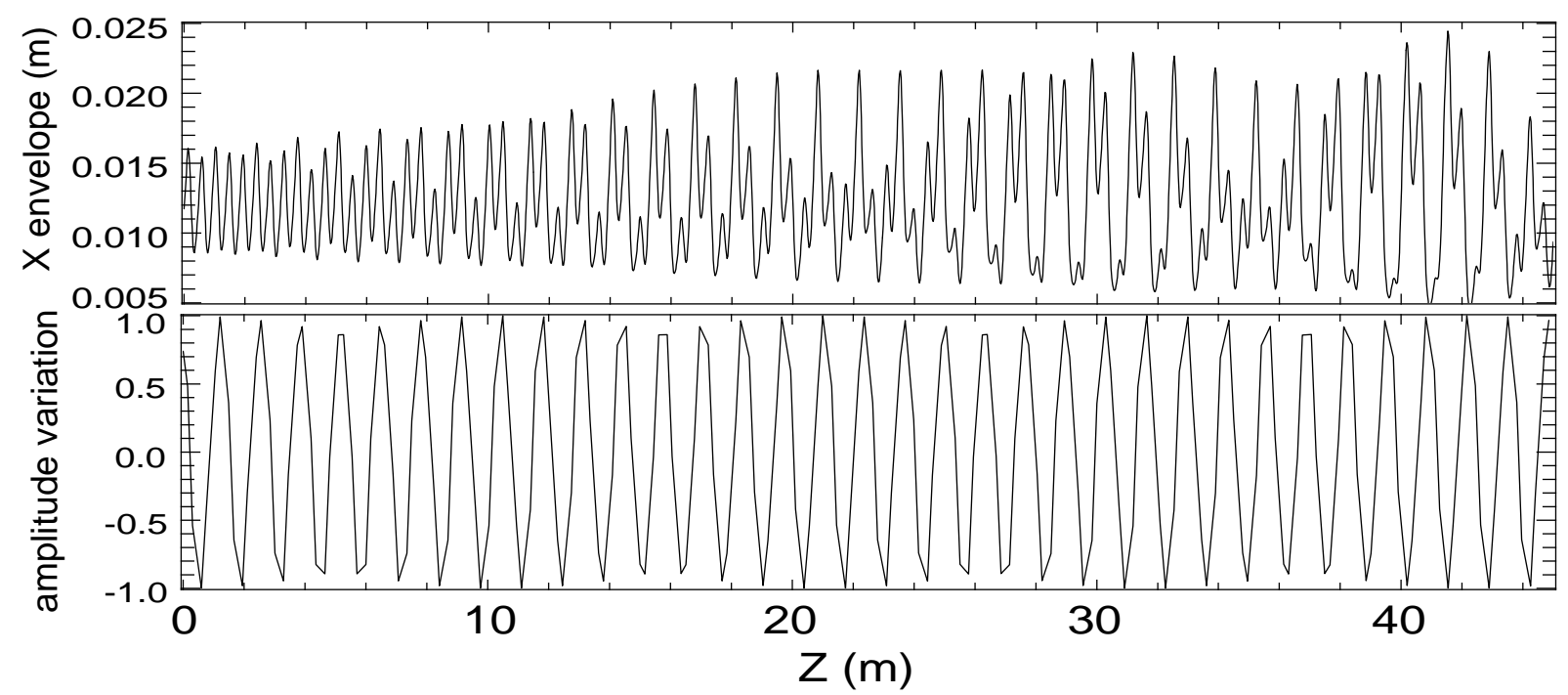

FIG. 14: Electron density departure from mean and beam envelope $x$ semi-axis (twice-RMS) versus axial position. The oscillations are approximately in phase until the envelope size becomes comparable with the pipe radius.

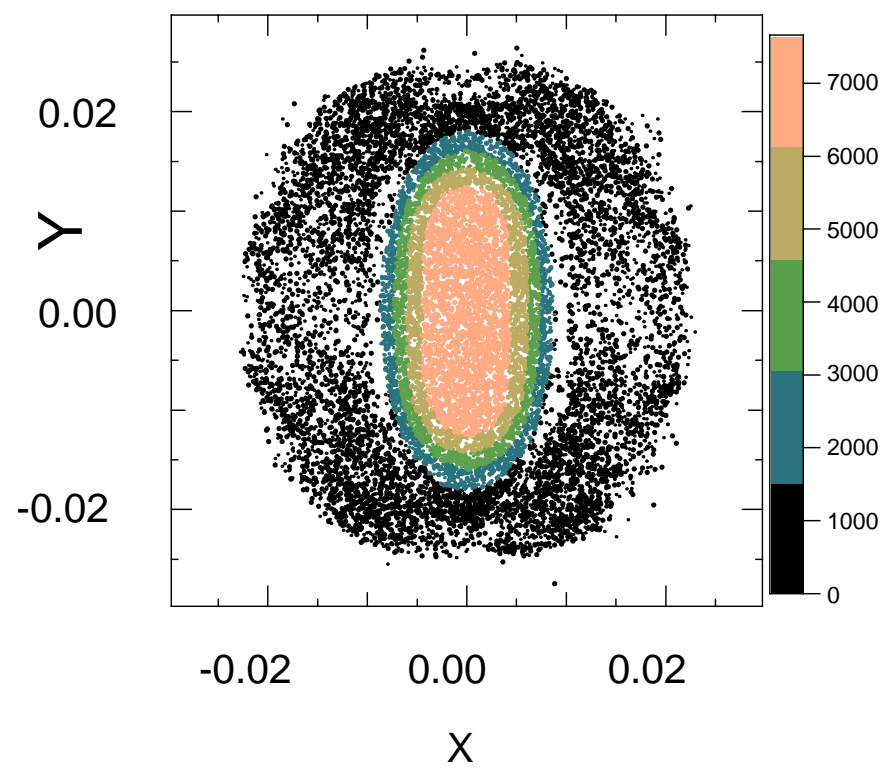

FIG. 15: $x-y$ scatter plot for random electron density perturbation; same as Fig. 3 but a later slice. 